\title{
Combining local and global evolutionary trajectories of brain- behavior equilibrium through game theory
}

- A novel prospective in integrative neuroscience -

\author{
Simone Di Plinio ${ }^{a, *}$, Sjoerd J H Ebisch ${ }^{a, b}$ \\ a Department of Neuroscience, Imaging, and Clinical Sciences, G D’Annunzio University of Chieti-Pescara, Chieti, Italy \\ b Institute for Advanced Biomedical Technologies (ITAB), G D’Annunzio University of Chieti Pescara, Chieti, Italy \\ *Corresponding author: simone.diplinio@unich.it
}

\begin{abstract}
The study of the evolution of brain-behavior relationships concerns understanding the causes and repercussions of cross- and within-species variability. Understanding such variability is a main objective of evolutionary and cognitive neuroscience, and it may help explaining the appearance of psychopathological phenotypes. Although the brain evolution is related to the progressive action of selection and adaptation through multiple paths (e.g., mosaic vs. concerted evolution, metabolic vs. structural and functional constraints), a coherent, integrative framework is needed to combine evolutionary paths and neuroscientific evidence. Here, we review the literature on evolutionary pressures focusing on structural-functional changes and developmental constraints. Taking advantage of progresses in neuroimaging and cognitive neuroscience, we propose a twofold model of brain evolution. Within this model, global and local trajectories imply rearrangements of neural subunits and subsystems as well as of behavioral repertoires of a species, respectively. We incorporate these two processes in a game in which the global trajectory shapes the structuralfunctional neural substrates (i.e., players), while the local trajectory shapes the behavioral repertoires (i.e., stochastic payoffs).
\end{abstract}

Keywords: Game theory; Graph theory; Evolution; Network; System; Brain-behavior relationships; Integrative neuroscience; Individual differences. 


\section{Introduction}

The evolution of the nervous system allowed higher fitness and adaptation of the species. An increase in the complexity of neural structures is observable in organisms with complex behavior, such as birds and mammals. In bilaterian organisms, ganglia tended to group anteriorly together with sensory organs (e.g., smell, sight) and to develop complex structures. Other possible structural configurations of the central nervous system in vertebrates would have been rather less advantageous and therefore never established. The nervous system has been subjected to both a continuous reconfiguration and expansion, which has contributed to the origin of more encephalized species. For example, in the hominin lineage, the total brain size first gradually increased (from 3 to 1.8 million of years ago; Falk et al., 2000) and then rapidly increased (from 600,000 to 150,000 years ago, Ruff et al., 1997) at least until the origins of modern humans (200,000 to 150,000 years ago, McDougall et al., 2005; Campbell and Tishkoff, 2008; Conard, 2010). This increase in size was accompanied by an important functional rearrangement through rewiring and structural reorganization (Deacon, 1997; Mesulam, 2000; Miller et al., 2019).

The expansion of the central nervous system, especially of the brain, underlies the development of more elaborated information processing as well as of a diversified and flexible pattern of behaviors (Schuppli and van Schaik, 2017; Whiten, 2017). As a consequence of expanded cognitive-behavioral repertoires, including complex social interactions (Dunbar and Shultz, 2007a) and multiple aspects of intelligence (Dunbar and Shultz, 2007b; Reader et al., 2011), the hominin lineage developed a human culture dependent upon both physiological and psychological-social evolution (Wilson, 1978; Laland, 2017). At present, the evolution of the interconnectedness between the physiological and behavioral dimensions has not been solved nor properly explored from a neuroscientific perspective. However, these processes are undoubtedly interdependent: structural and metabolic constraints (Hofman, 2014; Karbowski, 2014; Montgomery et al., 2017) conceivably shaped both the biological body plan of the species and the patterns of cognitive processes and behavioral responses available to individuals within the species.

Notwithstanding a general consensus about many evolutionary processes involving complex organisms (see Mayr, 2001; Gould, 2002; Laland et al., 2015), and despite informative research about brain-specific evolutionary pressures (Barton, 2000; Clark et al., 2001; de Winter and Oxnard, 2001; Song et al., 2013; Gomez-Robles et al., 2014), it is still unclear how the functional and structural features of the brain evolved in synergy with the cognitive abilities and behavioral 
predispositions of individuals within a species. For example, little has been theorized about which evolutionary pressures favored an irregular distribution of structural and functional features of the brain across individuals of the same species. These include, on one hand, structural features, such as cortical thickness, myelinization, and structural connectivity and, on the other hand, functional features, such as task-evoked activity and functional connectivity. Given the complications in integrating evolutionary, structural, functional, and behavioral specializations within the same framework (Bertossa, 2011; Feldman-Barrett and Satpute, 2013; Striedter, 2019, Llera et al., 2019), questions such as "what are the evolutionary causes of cross-species and within-species heterogeneity in the brain shape and functional organization?", "why is the within-species structural and functional variability inhomogeneous across brain's subunits and subsystems?", and "how is this physiological variability associated with behavioral variability?" are still unanswered.

In the current article, we propose a twofold model of the evolution of the brain-behavior relationship that outlines the root of both within-species and between-species variability of the brain. Firstly, we expand on the concepts of concerted vs. mosaic evolution (Finlay and Darlington, 1995; Barton and Harvey, 2000), functional and structural constraints (Montgomery et al., 2017), between-species variability (de Winter and Oxnard 2001; Gonda et al., 2013), and adaptation (Mayr, 2001; Laland et al., 2015), conceptualizing them as the backbones for long-lasting, speciesspecific changes in the neural architecture. We ascribe these structural-functional processes to a global trajectory of the evolution of the brain. Secondly, we suggest that the inter-individual, within-species variability in the main features of the brain architecture (de Winter and Oxnard 2001 ) is a short-term evolutionary instrument to yield adaptive changes in the frequencies and distributions of behavioral repertoires and cognitive abilities within populations. We ascribe these functional-behavioral processes to a local trajectory of the evolution of the brain. These two mechanisms relate to a species-specific structural-functional configuration and to an individualspecific functional-behavioral configuration, respectively, on which evolutionary pressures act differently. We identify the object of selection of these two trajectories in the following two different aspects of the phenotype: while the global trajectory mainly acts on structural-functional subunits (i.e., parcels; Glasser et al., 2016; Eickhoff et al., 2018) and subsystems (i.e., modules or subnetworks, Bullmore and Sporns, 2009; Rubinov and Sporns, 2010) of the brain, the object of selection for the local trajectory is the pattern of behavioral and social repertoires (Dunbar and Shultz, 2007a-b; Roughgarden, 2012).

In the final section of the paper, we propose a theoretical and methodological framework to 
investigate this brain-behavior configuration problem through the integration of game theory and graph theory in neuroscience. Since the global trajectory acts on a structural-functional, speciesspecific equilibrium, and the local trajectory acts on a functional-behavioral, individual-specific equilibrium, we identify the structural-functional features of the network (brain) as latent cost functions that determine the individual behavioral payoff pattern. The proposed approach could be applied to investigate both cross-species differences in the structure-function relationships (global trajectory) and within-species variability in brain-behavior relationships (local trajectory). We believe that bringing together evolutionary biology, network neuroscience, and cognitive science provides new theoretical and methodological possibilities to the study of the mind and brain.

We first provide a neuroscientific framework for the study of the brain, its evolution, and its relationship to behavior (section 2); we then present the local-global model of brain evolution (section 3), and we describe an approach aimed at elucidating brain-behavior relationships using game theory (section 4); we conclude our paper with some considerations on the model and its repercussions for future basic and clinical research (section 5).

\section{An integrative framework}

\subsection{Insights from evolutionary studies}

Two main contrasting models in evolutionary neurobiology have been proposed to explain the evolution of the brain and its subunits. The concerted model ascribes major events of covariation in brain structures to developmental events (Finlay and Darlington, 1995; Finlay et al., 2001), prevalently driven by a small number of genetic variations (Enard, 2016). Alternatively, the mosaic model relates localized divergences in brain structures to single functional roles (Barton and Harvey, 2000; Barton, 2007). Although these two models have often been considered as conflicting, they are both supported by scientific evidence. Moreover, it has been shown that the brain can evolve as a coordinate entity, and, concurrently, it can be exposed to localized modifications in both primates (Aldridge, 2011) and songbirds (Moore and DeVoogd, 2017).

Mosaic and concerted changes are not the only hypothesized drivers of brain evolution. Another, partially related point of view focuses on constraints, distinguishing between functional constraints and developmental constraints of the brain (Montgomery et al., 2017). Functional constraints would favor an allometric scaling between brain components (i.e., co-growth of functionally associated brain subunits) when they regulate similar adaptive behaviors (Barton and 
Harvey, 2000; Halley and Krubitzer, 2019). For example, if a specific subunit within a subsystem undergoes selection (i.e., evolves), then growth is promoted across the functionally connected brain regions, possibly reinforcing specialized behaviors. Instead, following the developmental constraints hypothesis, brain subunits tend to evolve together since selective pressures operate on developmental mechanisms (e.g., genetic expression) that equally affect all the subunits of a network (Charvet and Finlay, 2012; Workman et al., 2013).

A further separation emerges when considering between-orders or within-order divergences in brain morphology (de Winter and Oxnard, 2001; Gonda et al., 2013). In fact, while mosaic brain organization has been linked to selective adaptation of sub-orders (i.e., within-orders, Aristide et al., 2016), the between-orders comparison of brain morphology suggests an interplay between selective pressures and structural-metabolic constraints (Gomez-Robles et al., 2014), possibly depending on both mosaic and concerted mechanisms. Finally, although it is somewhat neglected, metabolic and structural constraints involve trade-offs that drive the biological configuration of the brain (Kardowski, 2014). Consequently, both microscale designs of cortical columns (Snider et al., 2010; Teeter and Stevens, 2011) and macroscale modular arrangements of brain subunits (Bassett et al., 2010; Chen et al., 2013) adhere to structural and physiological motifs both at the species level and at the individual level.

In summary, developmental, functional, and structural features of the nervous system are definitely intertwined to accomplish a species-specific brain-behavior equilibrium. However, an integrative view of evolutionary neurobiology and neuroscientific evidence that explains the role and weight of subunits (brain regions, structures) and subsystems (brain modules, subnetworks) in this equilibrium is still missing.

\subsection{Insights from neuroimaging}

Neuroimaging offers a powerful set of tools to investigate the differential involvement of brain subunits and subsystems in behavior. For example, prefrontal white matter seems to be a major mosaic contributor to evolutionary changes in primate brain reorganization (Smaers and Soligo, 2013). The contribution of prefrontal structures has also been linked to attentive and social skills (Powell et al., 2010; Asplund et al., 2010) and to abstract skills such as moral judgment and theory of mind (Forbes et al. 2010; Krienen et al., 2010). According to these studies, the prefrontal cortex is likely to have a significant importance in the inter-individual variability in brain-behavior interactions. However, cognitive abilities are more likely to be distributed over many brain units and subsystems (i.e., many subunits of the brain; Bathelt et al., 2019), that contribute to 
interindividual variability in brain-behavior interactions.

At the evolutionary level, macroscopic variations in the architecture of brain subunits (such as in the prefrontal cortex) are likely to derive from multiple circuital and genetic micro-variations with subsequent adaptation (DeFelipe, 2006, 2011). In neuroimaging studies, structural-functional subunits are called parcels and estimate surprisingly well structural and functional specializations (Glasser et al., 2016; Schaefer et al., 2018). Whereas highly reliable parcellation atlases are relatively recent, the idea of operational subunits in the brain is definitely less recent (Lorente de Nó, 1933). A more recent field of investigation is network neuroscience, which basically employs functional connectivity between brain subunits to model brain subsystems (Bassett and Sporns, 2017). Network neuroscience traditionally involves the analysis of co-activated regions (Hutchinson et al., 2013) but can also include other aspects of the brain, such as structural covariance (Anderson-Bloch and Finlay, 2013) or genomic patterns (Conaco et al., 2012; Valk et al., 2020). Through the implementation of graph theory in network neuroscience (Bullmore and Sporns, 2009; Rubinov and Sporns, 2010), neuroscientists can investigate the way ensembles of brain subunits functionally integrate or segregate during behavior (Sporns, 2013; Godwin et al., 2015), considering also structural features (Shi et al., 2019). Modern parcellations incorporate structural and functional aspects (e.g., cortical thickness, myelinization, brain activity, functional and structural connectivity) both at the regional level and at the modular level. This multimodality allows relating inter-individual differences in the brain architecture (at both subunit and subsystem levels) to the underlying behavioral and cognitive features (Eickhoff et al., 2018; Di Plinio and Ebisch, 2018; Bolt et al., 2020).

The lack of accurate definitions of brain subunits in the past hindered the study of specialized brain subunits in relation to evolutionary aspects, often constraining researchers to spurious subsampling techniques, cherry-picking regions of interest, or massive univariate corrections with consequent loss of statistical power. Thus, the development of an integrative theory on the evolution of the brain would greatly benefit from the implementation of reliable brain parcellations and the employment of multimodal methods. Nevertheless, it needs to be mentioned that the definition of brain subunits needs further elaboration, for example, because there is an intrinsic within-parcel variability (e.g., Jezzini et al., 2012; Bolognini et al., 2013; Bickart et al., 2014) and the resolution of parcellations can have multiple solutions (Schaefer et al., 2018).

\subsection{Insights from cognitive neuroscience}

Individual differences in psychological facets at cognitive and non-cognitive levels have been 
associated with diversified patterns of structural and functional brain features. For example, general cognitive abilities, like intelligence, have been associated with many graph measures such as structural covariance between fronto-parietal associative regions (Lerch et al., 2006), global efficiency (Li et al., 2009), white matter integrity (Kocevar et al., 2019), and global modularity in brain subsystems (Hilger et al., 2017). Similarly, general non-cognitive skills such as personality traits have been associated with both morphometric measures (Riccelli et al., 2017), global patterns of functional connectivity (Mulders et al., 2018), brain evoked activity (Kumari et al., 2004), cortical thickness (DeYoung et al., 2010), and structural covariance (Gardini et al., 2010) revealing that the variability in prefrontal, parietal, and superior temporal brain subunits is associated with personality traits. Furthermore, single brain structures can be associated with multiple behavioral and cognitive features. For example, activity of the amygdala is related to emotional interference in individual with avoidant personalities (Most et al., 2006), whereas its size covaries with both memory performance (Weniger et al., 2006) and social network extension (Bickart et al., 2011). Finally, single behavioral measures can be associated with functional architecture at multiple levels. In fact, we recently showed that variability in a specific cognitive index, such as intentional binding (a measure of the sense of agency), is simultaneously associated with graph measures at the subunit level in the supramarginal gyrus, at the subsystem level in a fronto-parietal module, and at the whole-brain level (Di Plinio et al., 2020).

A frequently neglected feature of the brain is that, since it has a baseline pattern of activity and connectivity (Gusnard and Raichle, 2001; Greicius et al., 2003), cognitive engagement not only produces regional increases in activity but also deactivations in the brain (i.e., regional decreases in activity compared to a baseline; Shulman et al., 1997; Fox et al., 2005). Thus, brain subunits may contribute both positively and negatively to behavior, possibly allowing higher performances during the task (Singh and Fawcett, 2008; Kelly et al., 2008; Hampson et al., 2010, Di Plinio et al., 2018). These characteristics of brain-behavior relationships illustrate the importance of a controlled allocation of metabolic resources to brain regions. Importantly, this equilibrium is taskspecific: the topographic patterns of task activations and deactivations are different across different tasks (Leech et al., 2014). These regional patterns of sparse activations/deactivations have probably been selected by evolution since they increase information exchange across brain subsystems (Bassett and Bullmore, 2016) and are metabolically advantageous as they maintain homeostasis (Hellyer et al., 2017) and limit inflammation due to overheating (Wang et al., 2014).

This evidence suggests that the variability in brain structure and function encodes behavioral 
and cognitive variability at overlapping hierarchic levels. Besides following structural and functional constraints, the brain's evolution has been driven by selective pressures aimed at optimizing the efficiency of the patterns of functional-behavioral variability across individuals of species, eventually producing a critical variability in the way individual brains can process information.

\subsection{Ad interim conclusions}

There is compelling evidence for a tight link between cross- and within-species reorganizations of the brain and behavioral variability. Cognitive and personality specializations are related to partially overlapping brain systems that developed over time favored by mosaic changes and by a constrained structural reorganization of the biological substrate. These subsystems must both be efficient and robust in order to face specific evolutionary pressures (Carrhuters, 2006) and to be robust against perturbations (Anderson and Finlay, 2014). However, the high inter-individual variability in brain geometry (Van Essen et al., 1984; Allen et al., 2006; Bakken et al., 2012; Song, 2013) suggests that other types of selective pressures may drive a parallel trajectory of the brain evolution related to differential cognitive and behavioral patterns within the same species (Hutchinson et al., 2013; Anderson-Bloch and Finlay, 2013). Even if cross-species and withinspecies patterns of structural and functional organizations have been used to explain behavioral specializations, single-individual behavioral specialization and species-level structural specializations have not yet been linked in a comprehensive way.

\section{A local-global model of the evolution of the brain}

\subsection{Defining local and global trajectories of brain evolution}

The mechanisms described above (i.e., between- vs. within-order evolution; developmental vs. functional processes hypothesis; mosaic vs concerted evolution; metabolic and structural constraints) have never been substantially unified. Here, we theorize a model covering two trajectories that shape(d) the evolution of the nervous system and of the mind. On one hand, we postulate a global trajectory that operates over large time intervals at the structural-functional level; on the other hand, a local trajectory operates in smaller time intervals, selecting the functional features of the brain and the associated behaviors of individuals within a species.

The global trajectory implies substantial functional and structural rearrangements of the central nervous system that persist over relatively long periods of time. Critical modification in the brain morphology occurs by gene duplication (Taylor and Raes, 2004; Johnson et al., 2009), modification to regulatory genetic pathways (Emes and Grant, 2012), brain pathway duplication 
(Chakraborty and Jarvis, 2015), modifications to specific coding sequences (Ono et al., 2014), or mRNA splice variants (Goymer, 2007; Gueroussov et al., 2015). Evolution along the global trajectory is also critically influenced by developmental mechanisms (Charvet and Striedter, 2011). These rearrangements impact species adaptation uniformly, that is, with almost the same result across all the individuals of the population. To give an example, the mutation of the gene FOXP2 in the hominin lineage (Enard et al., 2002) has been an important event for the evolution of language as well as other cognitive functions in humans (Fisher, 2017). This global evolutionary trajectory can be considered as a species-average, slow neural trajectory consisting of punctuated shifts and stasis periods and determined by natural selection, strong environmental changes, and speciation mechanisms. Thus, the global trajectory (i) acts on brain subunits, (ii) produces both concerted and mosaic rearrangements that change the structural or functional architecture of the species brain (also predisposing the development of selected brain subsystems), (iii) must comply with (and can modify) structural and developmental constraints (Gomez-Robles et al., 2014), (iv) is the main source of between-order evolution, and $(v)$ impacts both developmental and functional processes (Figure $1 \mathrm{~A}-\mathrm{B})$.
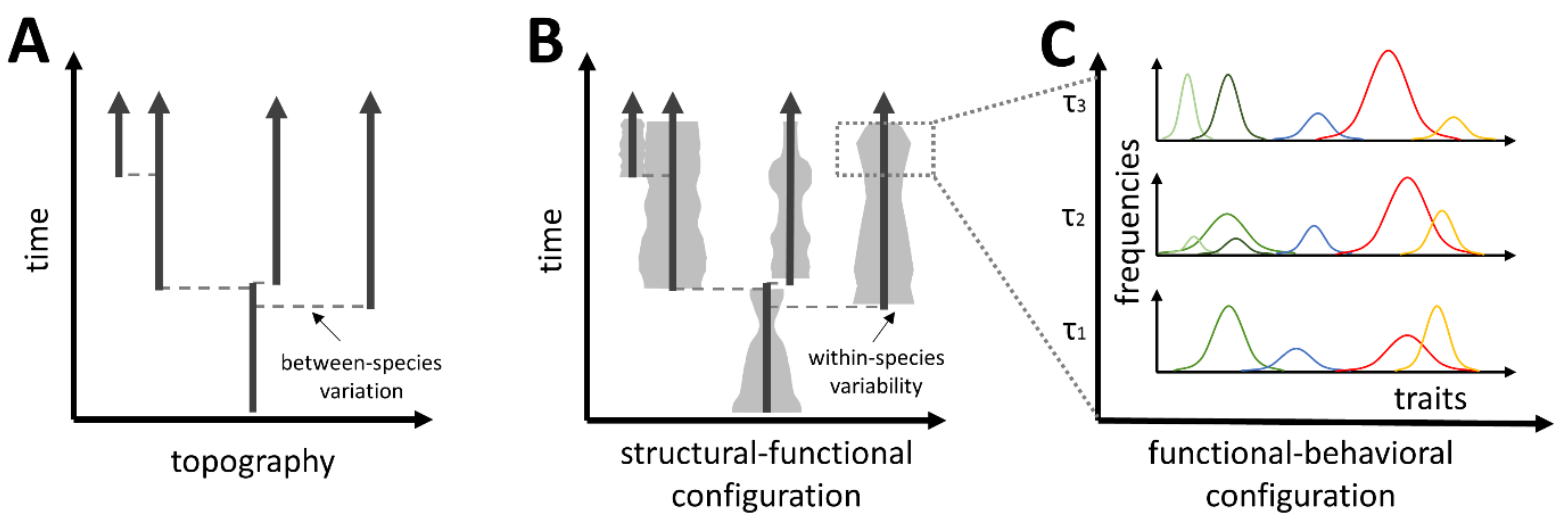

Figure 1. Global and local trajectories of brain evolution. (A) Following the punctuated equilibrium theory, the between-species differences are determined in critical periods in which speciation occurs; these periods are interspersed with cycles of relative stasis. (B) In the local-global model, the global trajectory includes punctuated equilibriums and speciation events, implying the persistence of a physiological variability across individuals (shaded colors). (C) In the subfigure, the frequency each latent trait is represented by a different color. The local trajectory of the evolution of the brain acts on the residual variability, redesigning the distribution of behavioral attitudes and cognitive skills (traits) over time in the population. Thus, in subsequent time intervals (e.g., from t1 to t2, t3, ...), the patterns of traits within the population changes and promote a flexible adaptation of the population to the environment (e.g., the red trait become more frequent, the green trait splits in two frequent sub-traits, the blue trait remains almost unchanged).

Within this global trajectory, we define a number of time intervals, $T$; each $\tau$ (with $\tau \in T$ ) encompasses the representation of the brain morphology (possible structural and functional patterns) of a species in a relatively short time interval. Within each time interval $\tau$, the interindividual, within-species variability is small enough to grant the robustness of selected phenotypes (i.e., stasis). Moreover, there is still a within-species physiological diversity that reflects 
and causes the cognitive-behavioral patterns of individuals within the species at time $\tau$. The residual physiological diversity is reflected by the differences in size, topography, connectional profiles, and functionality of the brain and its subunits across individuals.

The inter-individual diversity at time $\tau_{0}$ represents the substrate on which the local trajectory of the evolution of the brain operates, and the diversity at $\tau_{1}$ represents its own result. Rather than the mere increase in the frequency of a certain attribute within the population (i.e., natural selection and adaptation, e.g., FOXP2), selective pressures within local trajectories are represented by social selection (Roughgarden, 2012; Lyon and Montgomerie, 2012) and imply a redistribution of the frequencies and types of cognitive and behavioral profiles across consecutive time intervals (from $\tau_{0}$ to $\tau_{1}$ ). This redistribution supports an optimal range of adaptive and complementary phenotypes of individuals within a species. In other words, the inter-individual variability in the main features of the brain architecture is a short-term evolutionary strategy to support an appropriate diversification of behavioral attributes and cognitive abilities within populations (Figure 1C). To summarize, the local trajectory (i) acts on behavioral and psychological-social repertoires, (ii) produces both concerted and mosaic functional rearrangements linked to selective adaptation (Aristide et al., 2016), (iii) must comply with functional/metabolic constraints, (iv) is the main source of within-species brain evolution, and $(v)$ may directly impact functional processes (although it can indirectly impact structural aspects through plastic rearrangements; Pascual-Leone et al., 2005; Gallen and D’Esposito, 2019).

These two processes set up a neuro-evolutionary scheme that explains both inter-individual and inter-species variability in brain physiology and behavior. Both global and local trajectories are dependent on neo-Darwinian premises, such as the natural production of phenotypic variability, reciprocal organisms-environment causation, and selection (Mayr and Agassiz, 1999, Laland et al., 2015). However, since the object of selection (Mayr, 1997) for the local trajectory is the pattern of behavioral and social repertoires, this process is directly related to "soft" mechanisms, such as social selection and socio-cultural inheritance (Dunbar and Shultz, 2007; Roughgarden, 2012; Laland et al., 2015). Local and global trajectories drive the structural and functional organization of subunits in the species brain (global trajectory) and the inter-individual variability in brain functioning, which establishes the base for inter-individual differences in psychological-social features (local trajectory).

\subsection{Ad interim conclusions}

A consequence of the twofold model is that there are two simultaneous types of "equilibria" 
that coexist. The first equilibrium is related to the structural-functional relationships of the species brain, that is, its "average structural-functional architecture", or body plan. This equilibrium is quite stable as it must be robust and reflects developmental and structural constraints associated with both mosaic and concerted evolution (Finlay and Darlington, 1995; Barton and Harvey, 2000; Aldridge, 2011). Secondly, the local trajectory implies an evolving ensemble of traits, or variants within a species, and is practically unaffected by structural constraints. However, it is still influenced by functional and metabolic constraints that drive complex patterns of local mosaic variations and patterns of activations/deactivations in the brain associated with cognitive functions (Karbowski, 2014; Leech et al., 2014; Wang et al., 2014; Hellyer et al., 2017). The local component explains the inter-individual differences in stimulus-evoked brain activity and functional connectivity recorded in neuroscientific investigations, which entail different behavioral and cognitive attributes across individuals (Powell et al., 2010; Asplund et al., 2010; Forbes et al. 2010; Krienen et al., 2010; Bathelt et al., 2019). Modifying the functional-behavioral equilibria, the local trajectory affects the brain functioning on multiple levels, from the regional (brain subunits) to the modular (brain subsystems) to the whole-brain level (Bassett et al., 2010; Chen et al., 2013; Di Plinio et al., 2020). Finally, since this trajectory is primarily functional and is dominated by cognitive psychological-social selective pressures, it may putatively become stronger in more encephalized animals or in animals with more complex social interactions. The characteristics of the two trajectories are summarized in Figure 2.

These processes ensure the diversification of functional-behavioral profiles of individuals and provide a stochastic form of adaptability of individuals and of the species. As a consequence of evolutionary trajectories, the local diversification can deliver outlier profiles of brain-behavior relationships, eventually causing metabolic and connectional abnormalities that, in turn, may affect the mental health of the individual. In other words, psycho-pathological conditions may be promoted by excessive divergences from the brain-behavior equilibrium relationships deriving from extreme social pressures and the associated local evolutionary drift. For example, disconnections or over-connections in psychopathologies (Fornito et al., 2012; Ebisch and Aleman, 2016; Ćurčić-Blake et al., 2017; Romme et al., 2017) or in healthy individuals with a high predisposition to psychotic experiences (Di Plinio et al., 2020) can predispose the individual to clinical syndromes (van den Heuvel and Fornito, 2014; Waters and Jardri, 2015; Ćurčić-Blake et al., 2017; van den Heuvel et al., 2019). Besides fitting with the framework depicted above, this idea agrees with the interpretation of human behavioral-cognitive traits as different combinations of "positions" on continuous latent features that eventually caused the manifestation of pathological 
traits such as schizophrenia and related disorders (Insel et al., 2010; Morris and Cuthbert, 2012; van den Heuvel et al., 2019). However, this notion is also challenged by the belief that severe psychiatric disorders cannot be situated along continuous dimensions since they differ qualitatively (Ross and Margolis, 2018, 2019). From our point of view, evolutionary on/off switches can be best ascribed to a global trajectory of brain evolution, while multidimensional patterns of variability (including pathological variations) are the consequence of a local trajectory. In the next section, we provide a methodological framework that can stimulate future investigations on the topic.

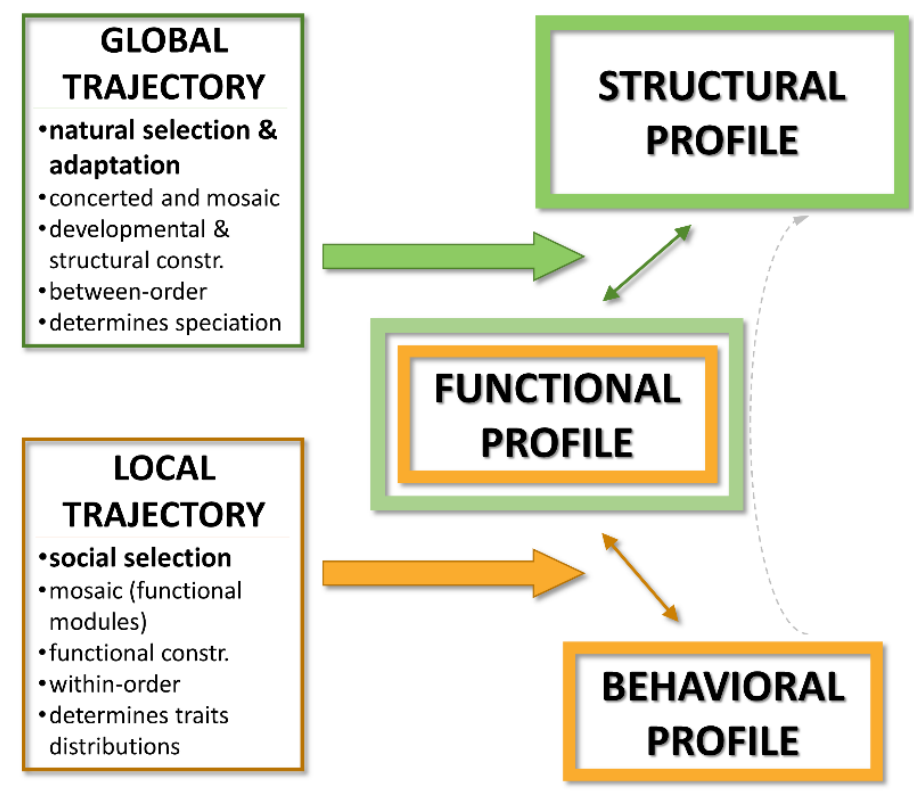

Figure 2. Schematic representation of local and global trajectories and their effect on the brain-behavior relationships.

\section{Evolving networks play games}

\subsection{Introducing game theory}

Game theory has been implemented in evolutionary studies to analyze stochastic adaptation in biological evolution (Metz et al., 1996), adaptability and stability underlying speciation (Geritz et al., 1998), evolutionary dynamics through graph theory (Lieberman et al., 2005), coevolution (Ripa et al., 2009), and extinction (Johansson and Dieckmann, 2009). Moreover, it has been argued that game theory could be an appropriate tool for understanding and modeling natural selection (Brown, 2016). In this section, we describe the implementation of game theory to integrate local and global evolutionary trajectories of the brain-behavior equilibrium. We hypothesize that brainbehavior relationships can be modeled as a game played by interconnected brain subunits in the brains of highly encephalized animals. We show that the joint implementation of game theory and graph theory would match the requirements for studying the dynamic organization of brain 
subunits and large-scale subsystems (i.e., modules or subnetworks), allowing proper crossindividuals and cross-species investigations. In game theory, players may choose between a finite number of actions. The outcome of their choice is called payoff and depends on the total pattern of actions (or strategies) adopted by players. In the classical example, called the prisoner's dilemma, the years in jail (payoff) of two prisoners (players) depends on their choice (action) to confess their crime or not (Figure 3A). Among the many variants of game theory, games can involve more than two players (n-players game; Nash, 1950; Czumaj et al., 2017), can be sequential or simultaneous depending on the temporal delay between choices (Fudenberg et al., 2014), can entail stochastic elements if the strategies or the payoff follow probabilistic laws (Shapley, 1953; Solan and Vieille, 2015), and can be cooperative or non-cooperative depending on whether or not players are allowed to form temporarily stable coalitions (Nash, 1951).

For the aims of this section, we consider the brain as a graph/network made up of interconnected subunits (parcels). The nodes of the network are represented by brain subunits (i.e., parcels), and the edges are the connectivity weights among subunits. Within this network, we define a game in which brain subunits (i.e., players) can cooperate in subsystems (i.e., coalitions). We introduce the concepts of structural profile, $\vec{\pi}$, structural state, $\vec{\Pi}$, functional profile, $\vec{\varphi}$, functional state, $\vec{\Phi}$, and behavioral profile, $\vec{\psi}$. The functional profile $\vec{\varphi}$ is defined for each single brain subunit and includes information about its functional connectivity profile (i.e., its connection with all the other parcels) and its evoked-activity profile (i.e., its degree of activation following virtually any type of stimuli). The functional state $\vec{\Phi}$ is the combination of all the functional profiles and thus holds the functional information about all the subunits of the brain. Similarly, the structural profile $\vec{\pi}$ is defined for each parcel and describes its structural characteristics (i.e., cortical thickness, geographic position in the brain, white matter connections), and the structural state $\vec{\Pi}$ is the combination of all the structural profiles. Finally, the behavioral profile $\vec{\psi}$ retains information about the individual's behavioral attributes (i.e., personality, temperament, and character traits) and its cognitive abilities (i.e., performance on virtually any task).

Thus, we theorize the brain as a network of players (brain parcels or subunits). Each player has unique structural characteristics $(\vec{\pi})$ and unique functional features, such as activations and functional connections $(\vec{\varphi})$ with other players, eventually forming dynamic coalitions. The functionality of the whole network, that is, the pattern of functional connectivity and brain activity in a specific environment, determines behavioral outcomes ( $\vec{\psi}$, Figure 3B). 


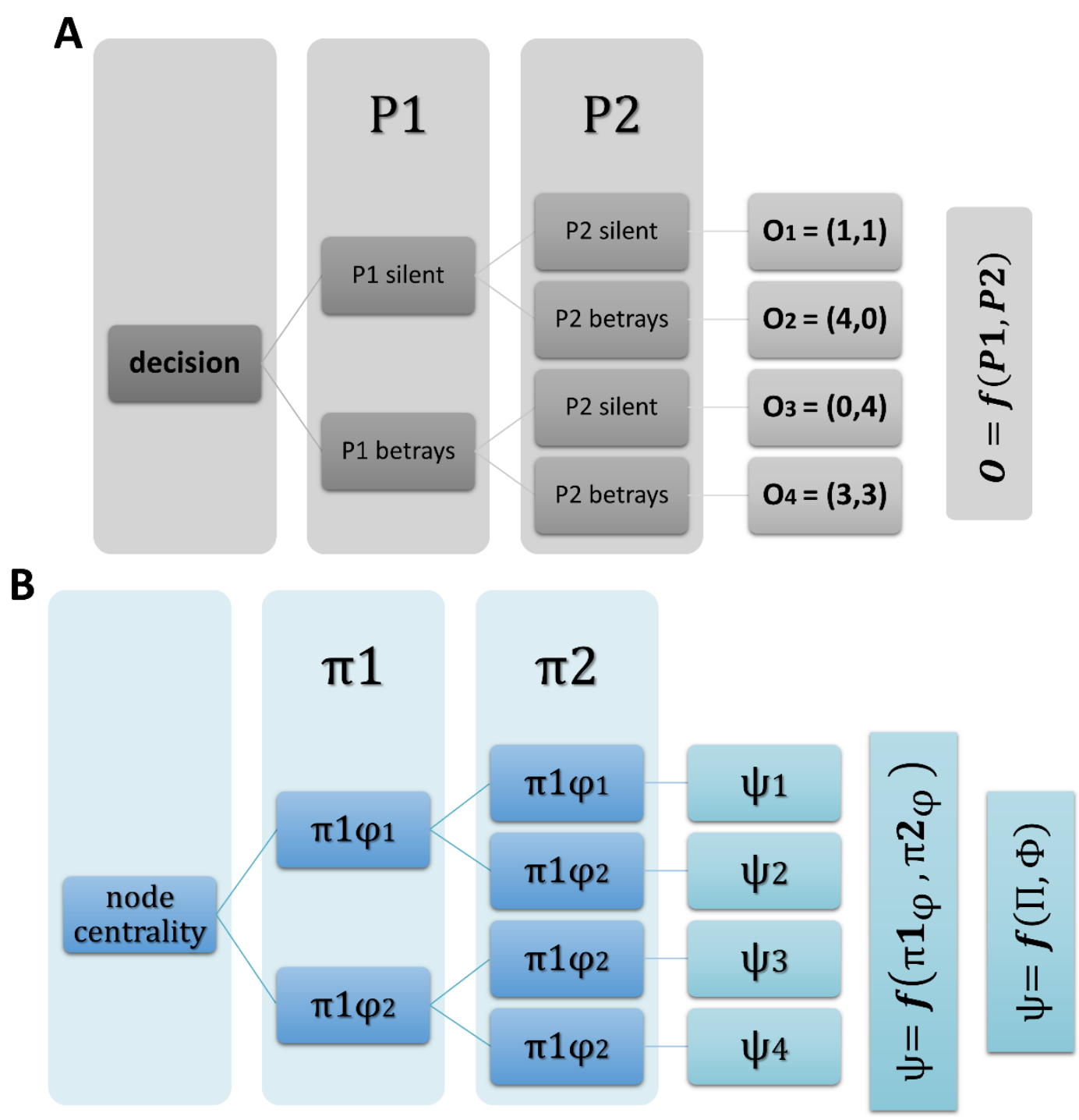

Figure 3. The extensive form of example games. (A) The prisoner's dilemma involves two players (P1, P2) and has four possible outcomes (O1-4) which represent the number of years in jail that the two prisoners will spend. (B) Example of a brain-game with two subunits ( $\pi 1$ and $\pi 2)$. In this example, each subunit can have two functional profiles $(\varphi)$. The combination of structural and functional elements imply the existence of $2 * 2=4$ possible behavioral outcomes $\left(\psi_{1-4}\right)$. Thus, the behavioral outcome is a function of the structural and functional states $(\Psi=\boldsymbol{f}(\Pi, \Phi)$ ).

\subsection{Linking games, graphs, and trajectories}

Selective pressures (see section 3) have two effects: on one hand, the global trajectory introduces variations that modify the structural-functional substrate, that is, they change the configuration of players in the game. On the other hand, the local trajectory rearranges the distribution of the functional states implemented in the network, thus determining a shift in the frequency of game rules across different networks (i.e., across different individuals). If we define as "state-space" the possible states of structural and functional features of a species brain, the whole set of individuals within a species can be displayed in the state-space in each time $\tau$. Across long 
time periods, evolution will shape the space of possible structural states $\vec{\Pi}$ and functional states $\vec{\Phi}$, changing the underlying biophysical fabric of the games. Over short time periods (e.g., from $\tau_{0}$ to $\tau_{1}$ ), evolution will shape the space of the available functional states $\vec{\Phi}$ and the consequential behavioral patterns $\psi$ by rearranging the distribution and frequencies of the payoffs, or cognitive/behavioral repertoires (Figure 4). In other words, the global trajectory of the evolution forges the pattern of available functional states and structural states (state-spaces). These states define the underlying game substrate (players), while the local trajectory shapes the distribution of functional and behavioral profiles within each species, defining the possible range of actions and payoffs of the game.

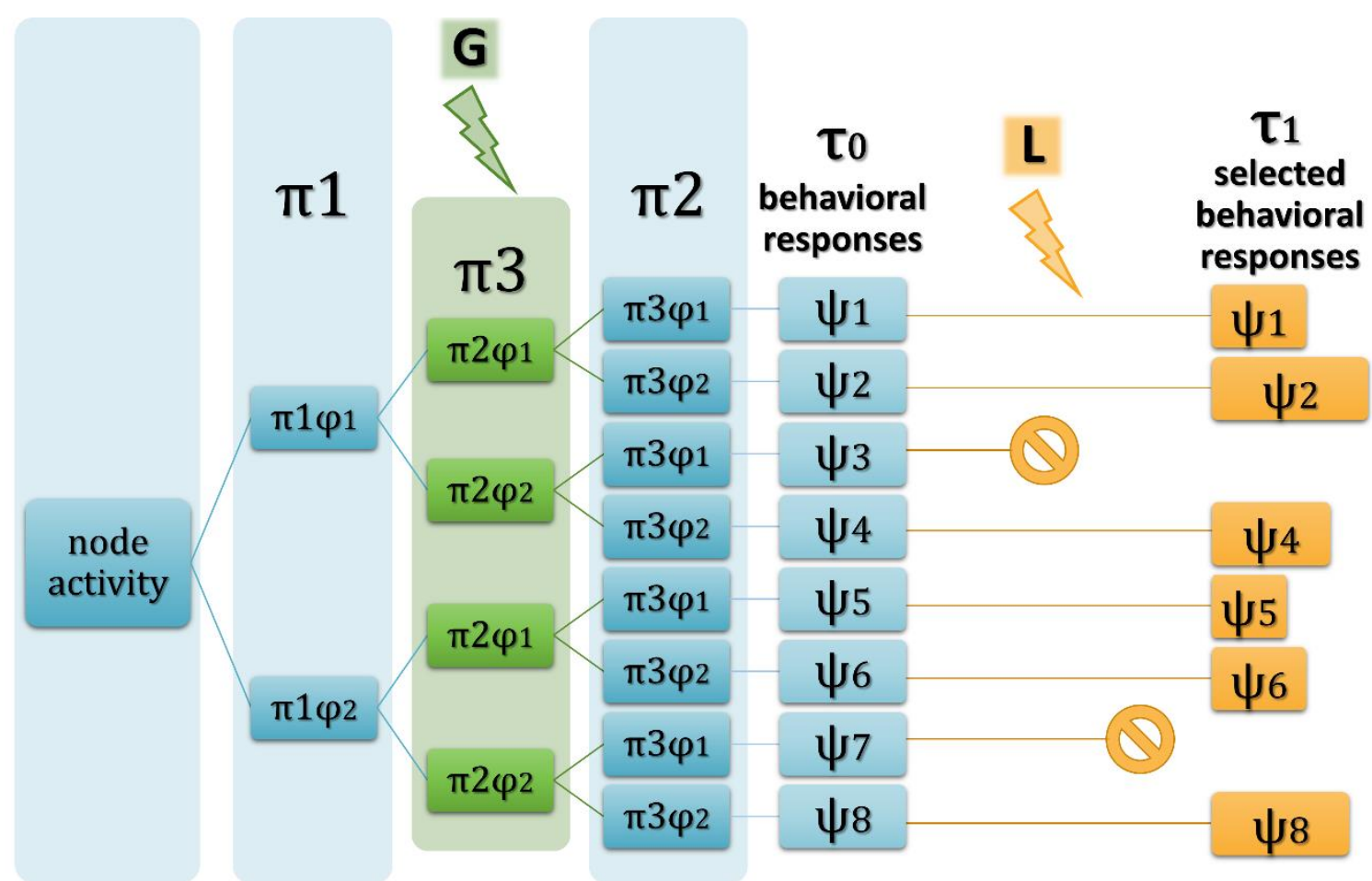

Figure 4. Effects of the global (G) and local (L) trajectories in and the brain-game. The global trajectory (green) changes the physiological substrate of the game (in this example, introducing a new processing unit, i.e., a player). The local trajectory (orange) changes the frequency and type of behavioral responses achievable through the brain functionality.

It is worth noting that this model accounts for the chronic and varying influence of the environment on behavior since we propose a dynamic game with stochastic payoffs. The environment-individual interactions imply probabilistic qualities of each feature within the behavioral profile. This means that the same state can induce different behaviors depending on environmental variables (Figure 5). It can be further observed that another possible cause of stochasticity in the actions may be the behavioral profile itself. For example, a behavioral trait (e.g., social anxiety) can influence behavior (e.g., accuracy during a task) only with specific environmental variables (e.g., if there are other conspecifics in the room). Moreover, the progress of the game is shaped by the outcome of the actions of the individual, involving relevant cognitive 
aspects in the game design. For example, the payoff can change following subtle reconfigurations of the players' choices over time. In other words, the behavioral performance can increase over time due to the reconfiguration of the underlying brain network structure, accounting for cognitive processes such as learning, adaptation, and habituation. These aspects, together with the dynamic properties of the game, may account for cross-species and within-species variability in brainbehavioral responses observed in neuroimaging studies. We would like to stress that the representation of games in Figures 3, 4, and 5 do not indicate constrained sequential processing of information. This representation, which is called "extensive form", is the only representation of a game that allows more than two dimensions and their stochastic payoffs to be represented in an interpretable way. The degree of consequentiality and directionality of elements in a graph can be ignored or can even be modeled using a priori information on temporal delays. This may further improve the biological validity of the model since the brain has a precise temporal structure in which some brain regions (such as the inferior parietal cortex and posterior cingulate cortex) are more likely to be drivers and other brain regions (such as the anterior prefrontal cortex) are often receivers (Deshpande et al., 2011; Majeed et al., 2011; Mitra et al., 2014).

Until this point, for clarity, we have described the payoff as a single outcome for the whole network that reflects behavioral or cognitive attributes. However, games usually associate a separate payoff with each player. In fact, assigning a single "whole-brain" payoff is unlikely and automatically shrinks the degrees of freedom. Thus, instead of a single behavioral, cognitive, or personality measure, the payoff should be a vector entailing the contribution of each brain subunit to behavior, that is, how much the behavioral outcome benefits from the functional profile of the parcel (i.e., from the player's strategy). This is particularly useful given that neuroimaging studies have showed that the contributions of a single brain region to a behavior can be positive, null, or negative depending on individual and environmental variables (Shulman et al., 1997; Fox et al., 2005; Singh and Fawcett, 2008; Hampson et al., 2010; Leech et al., 2014; Plinio et al., 2018). Nonetheless, it must be kept in mind that, the behavioral patterns at time $\tau_{1}$ would not necessarily include only states that produce a higher performance across cognitive domains with respect to $\tau_{0}$ because the selective pressures that drive the local trajectory are psychological-social and cultural. In other words, given that the evolution of a species does not automatically imply the acquisition of better skills over time, it should not be expected that the transition from $\tau_{0}$ to $\tau_{1}$ would necessarily improve all the cognitive abilities across all the individuals in the population. 

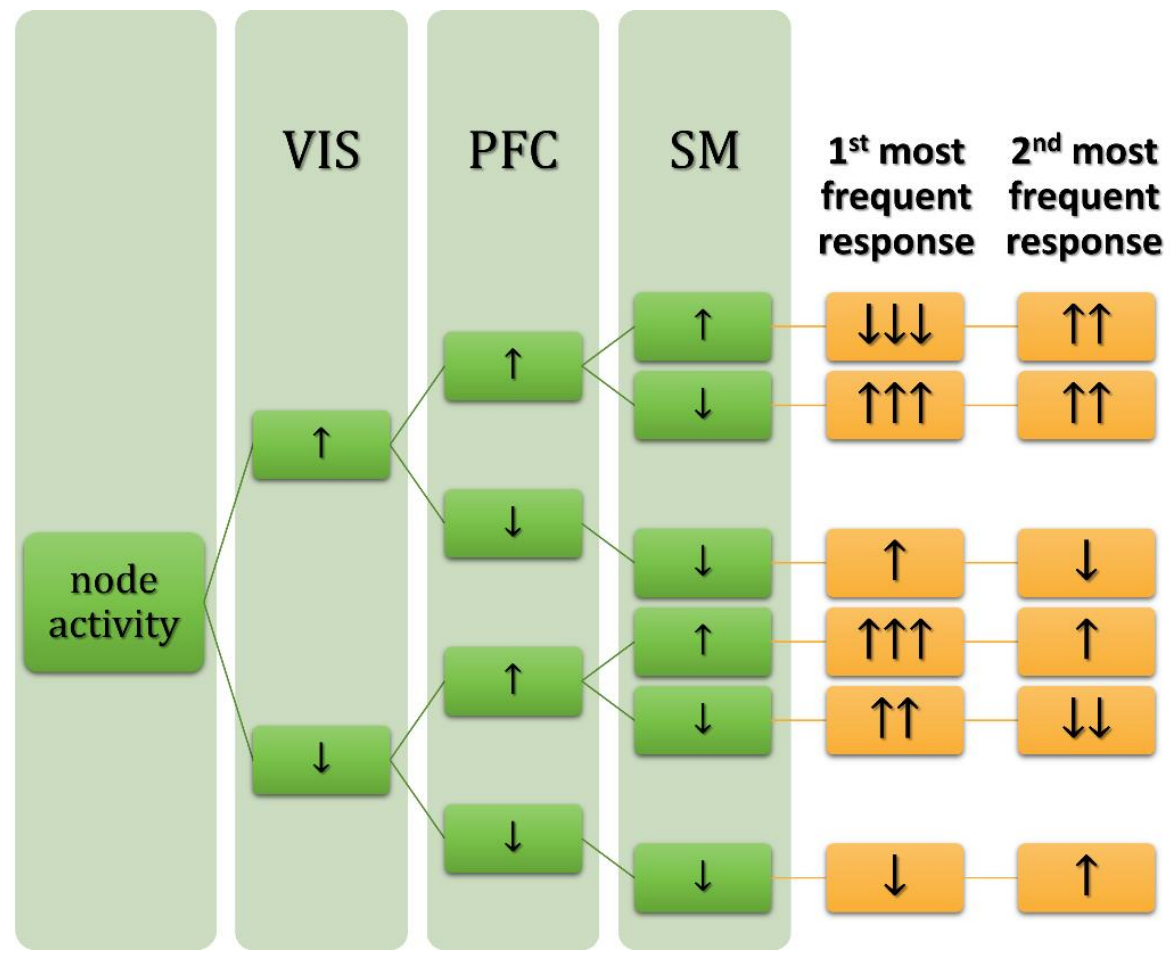

stochastic payoffs
(accuracy)
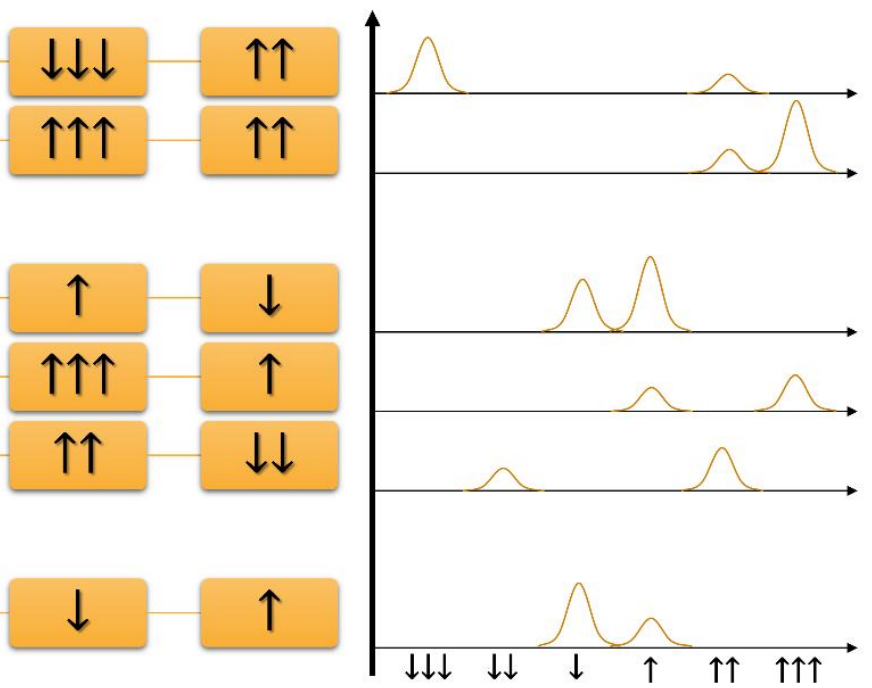

Figure 5. The extensive form of an example brain-game. In the example, there are three players: an input (visual subunit, VIS), an information processor (prefrontal cortex subunit, PFC), and an output (sensorimotor subunit, SM). The functional parameter considered is the task-evoked activity (for simplicity we omit the structural variability in this example). Each subunit can have two different strategies in the game, that is, its activity can increase $(\uparrow)$ or can decrease $(\downarrow)$. The stochastic payoff (behavioral responses) is represented by the pattern of behavioral responses selected by the local trajectory (cfr. Figure 4). It can be observed that a higher activation in PFC generally leads to better performances. However, the highest performance is observed in the configurations $\vec{\Phi}=\uparrow \uparrow \downarrow$ and $\vec{\Phi}=$ $\downarrow \uparrow \uparrow$, that is, when PFC activity is high but the whole system is not entirely active. In fact, when $\vec{\Phi}=\uparrow$ $\uparrow \uparrow$ the performance decreases, reflecting situations of overactivations in sensory cortices related to impaired performances occurring in psychopathological conditions. To note, for the sake of simplicity, we used a single measure to represent the payoff (accuracy). However, as we discussed above, we suggest that a more fitting payoff would be the contribution of each single brain subunit to the behavior.

\subsection{Ad Interim conclusions}

Modeling the brain network as a game is convenient and conspicuously fits with evolutionary processes and neuroscientific evidence, as follows:

- Global, long-term evolutionary processes change the underlying fundamental structure of the network in which the game develops, representing significant mosaic and concerted changes (Finlay and Darlington, 1995; Finlay et al., 2001; Barton and Harvey, 2000; Barton, 2007) and modifying the patterns of developmental and structural constraints (Barton and Harvey, 2000; Charvet and Finlay, 2012; Workman et al., 2013; Hofman, 2014; Karbowski, 2014; Montgomery et al., 2017; Halley and Krubitzer, 2019). 
- As the outcome is determined by the configuration of the whole network, behavior is determined by multivariate patterns of coactivation and inhibition among functionally related brain subunits, and not by the contribution of single brain subunit which instead represent sub-processors of chunks of information (Sporns, 2013; Godwin et al., 2015; Bassett and Sporns, 2017; Bathelt et al., 2019; Shi et al., 2019).

- Cooperative games take into account the dynamic contributions of players (i.e., the evolving behavioral outcome) as well as the dynamic formation of coalitions (i.e., subsystems or subnetworks) and their adjustment over time, mirroring cognitive processes such as learning, sensitization, and habituation (Squire and Wixted, 2011; Sheneman and Hintze, 2017).

- Evolutionary mechanisms of adaptation/exaptation (Mayr and Agassiz, 1999; Schuppli and van Schaik, 2017; Whiten, 2017; Laland, 2017) and evidence from neuroscientific studies suggest that the same behavior can be achieved through multiple physiological mechanisms. Borrowing a term from genetics, it can be stated that the structuralfunctional code of the brain for behavior is degenerate. Similarly, in the brain game, the same behavior can be achieved through multiple brain states.

- The suggested approach allows implementing highly reliable modern brain parcellations, multimodal imaging methods, and graph theory, possibly grounding future methodological development in integrative neuroscience.

A simplified model of the brain-game presented in this section is described by Figure 5. A final, relevant aspect of game theory is the existence of one or more combinations of strategies that generate(s) the best outcome for the entire network (equilibria; Nash, 1950, 1951; Ummels and Wojtczak, 2011). If we model the brain like a game, can we suppose that the evolutionary trajectories are pursuing one or more equilibria points? In other words, is the evolution of structural-functional features and behavioral correlates of a species brain a dynamic search for one or more locus of equilibrium? To date, answers to this question can only be speculative. We suggest that whatever the answer to this question is, the integration of game theory for investigating between-species and within-species variation in brain architecture may be rather revealing in addition to possibly favoring the development of new methodological and theoretical approaches.

\section{Summary and future directions}

The transition across different phenotypes is a universal evolutionary mechanism ascribable to the progressive action of selection (Darwin, 1859). Still, the backlashes of brain-specific 
evolutionary paths on the links between brain reorganization (Deacon, 1997; Mesulam, 2000; Mayr, 2001; Gould, 2002; Laland et al., 2015; Miller, 2019), human socio-cultural traits (Wilson, 1978; Dunbar and Shultz, 2007a; Laland, 2015), and behavioral complexity (Dunbar and Shultz, 2007b; Reader et al., 2011) is still unanswered, mainly due to the problematic integration of many branches of knowledge in the same scheme (Bertossa, 2011; Striedter, 2019; Llera et al., 2019). In the present paper, we unify these two distinct evolutionary mechanisms in a single model taking advantage of neuroscientific evidence, graph theory, and game theory.

The first trajectory of the brain evolution shaped by natural selection acts upon a biophysical object of selection: the structural-functional profile of the subunits of the brain (Lorente de Nó, 1933; Glasser et al., 2016; Eickhoff et al., 2018; Schaefer et al., 2018). These processes favor a substantial structural-functional reorganization of brain morphology (Finlay and Darlington, 1995; Finlay et al., 2001) and of its subunits (Barton and Harvey, 2000; Barton, 2007), representing a global trajectory of brain evolution. This trajectory impacts the species brain uniformly through modifications in genetic sequences and pathways (Taylor and Raes, 2004; Johnson et al., 2009; Emes and Grant, 2012; Ono et al., 2014; Gueroussov et al., 2015; Fisher, 2017) and thus may determine new developmental constraints (Charvet and Stridter, 2011). The global trajectory is, therefore, the main cause of cross-species variation in brain morphology (Metz et al., 1996; de Winter and Oxnard, 2001) and is directly related to speciation (Geritz et al., 1998). The second, complementary trajectory of the evolution of the brain is primarily shaped by social selection (Dunbar, 2009; Roughgarden, 2012; Lyon and Montgomerie, 2012) and acts upon a non-physical object of selection: the pattern of brain functional states and their associated behavioral repertoires (Dunbar and Shultz, 2007a-b; Riccelli et al., 2017; Mulders et al., 2018) and cognitive abilities (Lerch et al., 2006; Li et al., 2009; Hilger et al., 2017; Kocevar et al., 2019). This process represents a local trajectory of the evolution of the brain and supports the modulation of the frequencies and distributions of cognitive and behavioral profiles across consecutive time intervals and within individuals of the same species. Thus, the local trajectory yields adaptive changes overt short time periods while maintaining an equilibrium between adaptation and efficiency (Carrhuters, 2006; Anderson and Finlay, 2014) and, more generally, determining within-order variations (Van Essen et al., 1984; de Winter and Oxnard, 2001; Allen et al., 2006; Bakken et al., 2012; Song, 2013; Gonda et al., 2013).

We incorporated global and local trajectories into a single framework taking advantage of graph theory (Bullmore and Sporns, 2009; Rubinov and Sporns, 2010; Sporns, 2013; Godwin et al., 
2015; Bassett and Sporns, 2017; Shi et al., 2019) and game theory (Nash, 1950, 1951; Lieberman et al., 2005; Ripa et al., 2009; Johansson and Dieckmann, 2009; Brown, 2016). In the brain game, the global trajectory shapes the pattern of available structural-functional neural substrates of each species, while the local trajectory shapes the distribution of functional profiles supporting behavior, defining the stochastic (Shapley, 1953; Ummels and Wojtczak, 2011; Solan and Vieille, 2015) range of achievable payoffs, that is, the patterns of behavioral repertoires and cognitive skills of individuals within a species. We note that, even if we considered unprocessed features (e.g., raw brain activations, raw behavioral scores) in the brain-game model, we suggest that the best way to study brain-behavior associations is through the extraction of latent factors from physiological and psychological observations (Feldman-Barrett and Satpute, 2013; Di Plinio and Ebisch, 2018; Di Plinio et al., 2019, 2020; Bolt et al., 2020). The brain-game model encompasses structural (e.g., structural connectivity, myelinization, cortical thickness), functional (e.g., task-evoked activity, functional connectivity), behavioral (e.g., cognitive performance, personality traits), and evolutionary features in the same framework, modeling the brain both as a system and as a collection of cooperating subunits. Such model would also allow (i) cross- and within-species neuro-evolutionary investigations, (ii) the study of subject-level deviations from the trajectory/equilibrium as possible indicators of sub-optimal states that facilitate clinical conditions (Fornito et al., 2012; Ebisch and Aleman, 2016; Ćurčić-Blake et al., 2017; Romme et al., 2017; van den Heuvel et al., 2019), (iii) the detection of hot spots contributing to cognitive dimensions, that is, brain subunits whose features are critical for specific traits or pathologies.

In conclusion, we propose a first attempt to link neuro-evolutionary outlines through game theory and neuroscience-informed graphs. However, we expect this framework to be further improved in the near future. For example, our model is entirely quantitative, but functional units are likely to differ across individuals also qualitatively. In fact, learning new skills can shape specific cortical units by increasing their efficiency in information processing and, thus, increasing an individual's performance (Anderson and Finlay, 2013; Gallen and D'Esposito, 2019) without necessitating a macroscopic reconfiguration but only through modifications in the micro-circuitry of specific subunits (DeFelipe, 2006, 2011; Emes and Grant, 2012; Alexander-Bloch and Finlay, 2013). It should also be kept in mind that we did not consider the influence of non-brain physical attributes on the brain itself since we focused essentially on the brain. Finally, since the individual brain undergoes a very large number of state changes at the functional level (Pascual-Leone et al., 2005; Gallen and D'Esposito, 2019), the frequency and type of individual behavioral attitudes and cognitive abilities change over time (Squire and Wixted, 2011; Sheneman and Hintze, 2017). Thus, 
a sub-local trajectory of the brain evolution accounting for the developmental and plastic intraindividual evolution of brain-behavioral interactive patterns during the lifespan can be hypothesized. We expect local and sub-local trajectories to be more prominent in highly encephalized mammals given their dependence on social interactions and plasticity. Synthesizing these aspects to interpret brain-behavior relationships and their evolution poses a challenge for integrative neuroscience. 


\section{REFERENCES}

Aldridge, K. 2011. Patterns of differences in brain morphology in humans as compared to extant apes. J Hum Evol, 60, 94-105.

Alexander-Bloch, A, Giedd, JN, Bullmore, E. 2013. Imaging structural co-variance between human brain regions. Net Rev Neurosci, 14, 322-336.

Allen, JS, Bruss, J, Damasio, H. 2006. Looking for the lunate sulcus: A magnetic resonance imaging study in modern humans. Anat Rec A Discov Mol Cell Evol Biol, 288(8), 867-876.

Anderson, ML, Finlay, BL. 2014. Allocating structure to function: The strong links between neuroplasticity and natural selection. Front Hum Neurosci, 7(918), 1-16.

Aristide, L, dos Reis, SF, Machado, AC, Lima, I, Lopes, RT, Perez, SI. 2016. Brain shape convergence in the adaptive radiation of New World monkeys. Proc Natl Acad Sci, 113(8), 2158-2163.

Asplund, CL, Todd, JJ, Snyder, AP, Marois, R. 2010. A central role for the lateral prefrontal cortex in goal-directed and stimulus-driven attention. Nat Neurosci, 13, 507-514.

Bakken, TE, Roddey, JC, Djurovic, S, et al. 2012. Association of common genetic variants in GPCPD1 with scaling of visual cortical surface area in humans. Proc Natl Acad Sci, 109, 3985-3990.

Barton, RA, Harvey, Ph. 2000. Mosaic evolution of brain structure in mammals. Nature, 405(6790), 1055-1058.

Barton, RA. 2006. Primate brain evolution: Integrating comparative, neurophysiological, and ethological data. Evol Anthropol, 15(6), 224-236.

Barton, RA. 2012. Embodied cognitive evolution and the cerebellum. Phil Trans R Soc B, 367, 2097-2107.

Bassett, DS, Greenfield, DL, Meyer-Lindenberg, A, Weinberger, DR, Moore, SW, Bullmore, ET. 2010. Efficient physical embedding of topological complex information processing networks in brains and computer circuits. PLoS Comput Biol, 6, e1000748.

Bassett, DS, Bullmore, ET. 2016. Small-world brain networks revisited. Neuroscientist, 23(5), 499-516.

Bassett, DS, Sporns, O. 2017. Network neuroscience. Nat Neurosci, 20(3), 353-364.

Bathelt, J, Scerif, G, Nobre, AC, Astle, DE. 2019. Whole-brain white matter organization, intelligence, and educational attainment. Trends Neurosci Educ, 15, 38-47.

Bertossa, RC. 2011. Morphology and behavior: Functional links in development and evolution. Phil Trans R Soc Lond B, 366, 2056-2068.

Bickart, KC, Wright, Cl, Dautoff, RJ, Dickerson, BC, Barrett, LF. 2011. Amygdala volume and social network size in humans. Nat Neurosci, 14, 163-164.

Bickart, KC, Dickerson, BC, Barrett, LF. 2014. The amygdala as a hub in brain networks that support social life. Neuropsychologia, 63, 235-248.

Bolognini, N, Convento, S, Rossetti, A, Merabet, LB. 2013. Multisensory processing after a brain damage: Clues on post-injury crossmodal plasticity from neuropsychology. Neurosci Biobehav Rev, 37(3), 269-278.

Bolt, T, Nomi, JS, Arens, R, Vij, SG, Riedel, M, Salo, T, Laird, AR, Eickhoff, SB, Uddin, LQ. 2020. Ontological dimensions of cognitive-neural mappings. Neuroinformatics, DOI: 10.1007/s12021-020-09454-y.

Brown, JS. 2016. Why Darwin would have loved evolutionary game theory. Proc R Soc B, 283, 20160847.

Bullmore, E, Sporns, O. 2009. Complex brain networks: Graph theoretical analysis of structural and functional systems. Nat Rev Neurosci, 10(3), 186-198.

Campbell, MC, Tishkoff, SA, 2008. African genetic diversity: Implications for human demographic history, modern human origins, and complex disease mapping. Annu Rev Genomics Hum Genet, 9, 403-409.

Carrhuters, P. 2006. The architecture of the mind: Massive modularity and the flexibility of thought. Oxford Clarendon Press.

Chakraborty, M, Jarvis, ED. 2015. Brain evolution by pathway duplication. Phil Trans R Soc B, 370(20150056).

Charvet, CJ, Striedter, GF. 2011. Developmental modes and developmental mechanisms can channel brain evolution. Front Neuroanat, 5(4), 1-5.

Chavret, CJ, Finlay, BL. 2012. Embracing covariation in brain evolution: Large brains, extended development, and flexible primate social systems. Prog Brain Res, 195, 71-87.

Chen, Y, Wang, S, Hilgetag, CC, Zhou, C. 2013. Trade-off between multiple constraints enables simultaneous formation of modules and hubs in neural systems. PLoS Comput Biol, 9, e10002937.

Clark, DA, Mitra, PP, Wang, SS. 2001. Scalable architecture in mammalian brains. Nature, 411, 189-193. 
Conaco, C, Bassett, DS, Zhou, H, Arcila, ML, Degnan, SM, Degnan, BM, Kosik, KS. 2012. Functionalization of a protosynaptic gene expression network. Proc Natl Acad Sci, 109(1), 10612-10618.

Conard, NJ. 2010. Cultural modernity, consensus or conundrum? Proc Natl Acad Sci, 107, 7621-7622.

Ćurčić-Blake, B, Ford, JM, Hubl, D, Orlov, ND, Sommer, IE, Waters, F, Allen, P, Jardri, R, Woodruff, PW, David, O, Mulert, C, Woodward, TS, Aleman, A. 2017. Interactions of language, auditory and memory brain networks in auditory verbal hallucinations. Prog Neurobiol, 148, 1-20.

Darwin, CR. 1859. On the origin of species. Cambridge: Harvard University Press.

Deacon, DW. 1997. What makes the human brain different? Ann Rev Anthropol, 26, 337-357.

de Winter, W, Oxnard, CE. 2001. Evolutionary radiations and convergences in the structural organization of mammalian brains. Nature, 409, 710-714.

Deshpande, G, Santhanam, P, Hu, X. 2011. Instantaneous and causal connectivity in resting state brain networks derived from functional MRI data. Neurolmage, 54, 1043.1052.

DeYoung, CG, Hirsh, JB, Shane, MS, Papademetris, X, Rajeevan, N, Gray, JR. 2010. Testing predictions from personality neuroscience: Brain structure and the Big five. Psychol Sci, 21(6), 820-826.

Di Plinio, S, Ferri, F, Marzetti, L, Romani, GL, Northoff, G, Pizzella, V. 2018. Functional connections between activated and deactivated brain regions mediate emotional interference during externally directed cognition. Hum Brain Mapp, 39, 3579-3610.

Di Plinio, S, Ebisch, SJH. 2018. Brain network profiling defines functionally specialized cortical networks. Hum Brain Mapp, 39(18), 1-18.

Di Plinio, S, Arnò, S, Perrucci, MG, Ebisch, SJH. 2019. Environmental control and psychosis-relevant traits modulate the prospective sense of agency in non-clinical individuals. Consc Cogn, 73, 102776.

Di Plinio, S, Perrucci, MG, Aleman, A, Ebisch, SJH. 2020. I am Me: Brain systems integrate and segregate to establish a multidimensional sense of self. Neurolmage, 205 (116284).

Dunbar, RIM, Shultz, S. 2007a. Understanding primate brain evolution. Phil Trans R Soc B, 362, 649-658.

Dunbar, RIM, Shultz, S. 2007b. Evolution in the social brain. Science, 317, 1344-1347.

Dunbar, RIM. 2009. The social brain hypothesis and its implication for social evolution. Ann Hum Biol, 36(5), 562572.

Ebisch, SJH, Perrucci, MG, Ferretti, A, Del Gratta, C, Romani, GL, Gallese, V. 2008. The sense of touch: Embodied simulation in a visuotactile mirroring mechanism for observed animate or inanimate touch. J Cogn Neurosci, 20(9), 1611-1623.

Eickhoff, SB, Constable, RT, Yeo, BTT. 2018. Topographic organization of the cerebral cortex and brain cartography. Neurolmage, 170, 332-347.

Emes, RD, Grant, SG. 2012. Evolution of synapse complexity and diversity. Annu Rev Neurosci, 35, 111-131.

Enard, W, Przeworski, M, Fisher, SE, Lai, CS, Weibe, W, Kitano, T, Monaco, AP, Pääbo, S. 2002. Molecular evolution of FOXP2, a gene involved in speech and language. Nature, 418(6900), 869-872.

Enard, W. 2016. The molecular basis of human brain evolution. Curr Biol, 26(20), R1109-R1117.

Falk, D, Redmond Jr, JC, Guyer, J, Conroy, C, Recheis, W, Weber, GW, Seidler, H. 2000. Early hominid brain evolution: A new look at old endocasts. J Hum Evol, 38, 695-717.

Finlay, BL, Darlington, RB. 1995. Linked regularities in the development and evolution of mammalian brains. Science, 268, 1578-1584.

Finlay, BL, Darlington, RB, Nicastro, N. 2001. Development structure in brain evolution. Behav Brain Sci, 24(2), 263278.

Fisher, SE, Marcus, GF. 2006. The eloquent ape: Genes, brains, and the evolution of language. Nat Rev Genet, 7, 920.

Fisher, SE. 2017. Evolution of language, lessons from the genome. Psychon Bull Rev, 24(1), 34-40.

Forbes, CE, Grafman, J. 2010. The role of human prefrontal cortex in social cognition and moral judgement. Annu Rev Neurosci, 33, 299-324.

Fornito, A, Zalesky, A, Pantelis, C, Bullmore, ET. 2012. Schizophrenia, neuroimaging, and connectomics. Neurolmage, 62(4), 2296-2314.

Fudenberg, D, Yuhta, I, Kominers, SD. 2014. Delayed-response strategies in repeated games with observation lags. J Econ Theory, 150(1), 487-514.

Gallen, CL, D’Esposito, M. 2019. Brain modularity: A biomarker of intervention-related plasticity. Trends Cogn Sci, 
23(4), 293-304.

Gardini, S, Cloninger, CR, Venneri, A. 2009. Individual differences in personality traits reflect structural variance in specific brain regions. Brain Res Bull, 79(5), 265-270.

Geritz, SA, Meszéna, G, Metz, JA. 1998. Evolutionarily singular strategies and the adaptive growth and branching of the evolutionary tree. Evol Ecol, 12, 35-57.

Glasser, MF, Coalson, TS, Robinson, EC, Hacker, CD, Harwell, J, Yacoub, E, Ugurbil, K, Andersson, J, Beckmann, CF, Jenkinson, M, Smith, SM, Van Essen, DC. 2016. A multi-modal parcellation of human cerebral cortex. Nature, 536(6715), 171-178.

Godwin, D, Barry, RL, Marois, R. 2015. Breakdown of the brain's functional network modularity with awareness. Proc Natl Acad Sci, 112(12), 3799-3804.

Gomez-Robles, A, Hopkins, WD, Sherwood, CC. 2014. Modular structure facilitates mosaic evolution of the brain in chimpanzees and humans. Nat Comm, 5(4469).

Gonda, A, Herczeg, G, Merilä, J. 2013. Evolutionary ecology of intraspecific brain size variation: A review. Ecol Evol, 3(8), 2751-2764.

Goymer, P. 2007. Alternative splicing switches on the brain. Nat Rev Neurosci, 8, 576.

Gould, SJ. 2002. The structure of the evolutionary theory. Harvard University Press.

Greicius, MD, Krasnow, B, Reiss, AL, Menon, V. 2003. Functional connectivity in the resting brain: A network analysis of the default mode hypothesis. Proc Natl Acad Sci, 100(1), 253-258.

Gueroussov, S, gonatopoulos-Pornatzis, T, Irimia, M, Raj, B, Lin, ZY, Gingras, AC, Blencowe, BJ. 2015. RNA splicing. An alternative splicing event amplifies evolutionary differences between vertebrates. Science, 349, 868-873.

Gusnard, DA, Raichle, ME. 2001. Searching for a baseline: Functional imaging and the resting human brain. Nat Rev Neurosci, 2(10), 685-694.

Halley, AC, Krubitzer, L. 2019. Not all cortical expansions are the same: The coevolution of the neocortex and the dorsal thalamus in mammals. Curr Opin Neurobiol, 56, 78-86.

Hampson, M, Driedsen, N, Roth, JK, Gore, JC, Constable, RT. 2010. Functional connectivity between task-positive and task-negative brain areas and its relation to working memory performance. Magn Reason Imaging, 28(8), 1051-1057.

Hellyer, PJ, Clopath, C, Kehagia, AA, Turkheimer, FE, Leech, R. 2017. From homeostasis to behavior: Balanced activity in an exploration of embodied dynamic enviconmental-neural interaction- PLoS Comput Biol, 13(8), e1005721.

Hilger, K, Hekman, K, Fieman, CJ, Basten, U. 2017. Intelligence is associated with the modular structure of intrinsic brain networks. Sci Rep, 7(16088), 1-12.

Hofman, MA. 2014. Evolution of the human brain: When bigger is better. Frontiers in Neuroanatomy, 8(15), 1-12.

Hutchinson, RM, Womelsdorf, T, Allen, EA, Bandettini, PA, Calhoun, VD, Corbetta, M, et al. 2013. Dynamic functional connectivity: Promise, issues, and interpretations. Neurolmage, 80, 360-378.

Insel, T, Cuthbert, B, Garvey, M, Heinssen, R, Pine, DS, Quinn, K, et al. 2010. Research domain criteria (RDoC): Toward a new classification framework for research on mental disorders. Am J Psychiatry, 167(7), 748-751.

Jezzini, A, Caruana, F, Stoianov, I, Gallese, V, Rizzolatti, G. 2012. Functional organization of the insula and inner perisylvian regions. Proc Natl Acad Sci, 109(25), 10077-10082.

Johansson, J, Dieckmann, U. 2009. Evolutionary responses of communities to extinction. Evol Ecol Res, 11, 561588.

Johnson, R, Samuel, J, Ng, CK, Jauch, R, Stanton, LW, Wood, IC. 2009. Evolution of the vertebrate gene regulatory network controlled by the transcriptional repressor REST. Mol Biol Evol, 26, 1491-1507.

Karbowski, J. 2014. Constancy and trade-offs in the neuroanatomical and metabolic design of the cerebral cortex. Front Neural Circuit, 8(9), 1-16.

Kelly, AM, Uddin, LQ, Biswal, BB, Castellanos, FX, Milham, MP. 2008. Competition between functional brain networks mediates behavioral variability. Neurolmage, 39, 527-537.

Kocevar, G, Suprano, I, Stamile, C, Hannoun, S, Fourneret, P, Revol, O, Nusbaum, F, Sappey-Marinier, D. 2019. Brain structural connectivity correlates with fluid intelligence in children: A DTI graph analysis. Intelligence, 72, 6775.

Krienen, FM, Tu, P-C, Buckner, RL. 2010. Clan mentality: Evidence that the medial prefrontal cortex responds to close others. J Neurosci, 30(41), 13906-13915.

Kumari, V, Ffytche, DH, Williams, SCR, Gray, JA. 2004. Personality predicts brain responses to cognitive demands. J 
Neurosci, 24(47), 10636-10641.

Laland, KN, Uller, T, Feldman, MW, Sterelny, K, Muller, GB, Moczek, A, Jablonka, E, Odling-Smee, J. 2015. The extended evolutionary synthesis: Its structure, assumptions and predictions. Proc R Soc B, 282, 20151019.

Laland, KN. 2017. Darwin's unfinished symphony: How culture made the human mind. Princeton University Press. Leech, R, Scott, G, Carhart-Harris, R, Turkheimer, F, Taylor-Robinson, SD, Sharp, DH. 2014. Spatial dependencies between large-scale brain networks. PLoS One, 9(6), e98500.

Lerch, P, Worsley, K, Shaw, WP, Greenstein, DK, Lenroot, RK, Giedd, J, Evans, AC. 2006. Mapping anatomical correlations across cerebral cortex (MACACC) using cortical thickness from MRI. Neurolmage, 31, 993-1003.

Li, Y, Liu, Y, Li, J, Qin, W, Li, K, Yu, C, Jiang, T. 2009. Brain anatomical network and intelligence. PLoS Comput Biol, 5(5), e1000395.

Lieberman, E, Hauert, C, Nowak, MA. 2005. Evolutionary dynamics on graphs. Nature, 433(70239, 312-316.

Llera, A, Wolfers, T, Mulders, P, Beckmann, CF. 2019. Inter-individual differences in human brain structure and morphology link to variation in demographics and behavior. Elife, 8, e44443.

Lyon, BR, Montgomerie, R. 2012. Sexual selection is a form of social selection. Phil Trans R Soc B, 367, 2266-2273.

Majeed, W, Magnuson, M, Hasenkamp, W, Schwarb, H, Schumacher, EH, Barsalou, L, Keilholz, SD. 2011. Spatiotemporal dynamics of low frequency BOLD fluctuations in rats and humans. Neurolmage, 54(2), 11401150.

Margulies, DS, Ghosh, SS, Goulas, A, Falkiewicz, M, Huntenburg, JM. 2016. Situating the default-mode network along a principal gradient of macroscale cortical organization. Proc Natl Acad Sci, 113(44), 12574-12579.

Mayr, E. 1997. The objects of selection. Proc Natl Acad Sci, 94(6), 2091-2094.

Mayr, E, Agassiz, A. 1999. Understanding evolution. Trends Ecol Evol, 14, 372-373.

Mayr, E. 2001. The philosophical foundations of Darwinism. Proc Am Phil Soc, 145, 488-495.

McDougall, I, Brown, FH, Fleagle, JG. 2005. Stratigraphic placement and age of modern humans from Kibish, Ethiopia. Nature, 433, 733-736.

Mesulam, M. 2000. Brain, mind, and the evolution of connectivity. Brain Cogn, 42, 4-6.

Metz, JAJ, Geritz, SAH, Meszéna, G, Jacobs, FJA, van Heerwaarden, JS. 1996. Adaptive dynamics, a geometrical study of the consequences of nearly faithful reproduction. In Stochastic and Spatial Structures of Dynamic Systems. Amsterdam, The Netherlands.

Miller, IF, Barton, RA, Nunn, CL. 2019. Quantitative uniqueness of human brain evolution revealed through phylogenetic comparative analysis. Elife, 8, e41250.

Mitra, A, Snyder, AZ, Hacker, CD, Raichle, ME. 2014. Lag structure in resting-satte fMRI. J Neurophysiol, 111, 23742391.

Montgomery, SH, Mundy, NU, Barton, RA. 2017. Brain evolution and development: Adaptation, allometry and constraint. Proc Biol Sci, 283(1838).

Moore, JW, DeVoogd, TJ. 2017. Concerted and mosaic evolution of functional modules in songbird brains. Proc $R$ Soc B, 284(20170469).

Morris, SE, Cuthbert, BN. 2012. Research domain criteria: Cognitive systems, neural circuits, and dimensions of behavior. Dialogues Clin Neurosci, 14(1), 29-37.

Most, SB, Chun, MM, Johnson, MR, Kiehl, KA. 2006. Attentional modulation of the amygdala varies with personality. Neurolmage, 31(2), 934-944.

Mulders, P, Llera, A, Tendolkar, I, van Eijjndhoven, P, Beckmann, C. 2018. Personality profiles are associated with functional brain networks related to cognition and emotion. Sci Rep, 8(1), 13874.

Nash, J. 1950. Equilibrium points in N-Person games. Proc Natl Acad Sci, 36, 48-49.

Nash, J. 1951. Non-cooperative games. Ann Math, 54(4), 286-295.

Ono, H, Kozmik, Z, Yu, JK, Wada, h. 2014. A novel N-terminal motif is responsible for the evolution of neural crestspecific regulatory activity in vertebrate FoxD3. Dev Biol, 385, 396-404.

Pascual-Leone, A, Amedi, A, Fregni, F, Merabet, LB. 2005. The plastic human brain cortex. Annu Rev Neurosci, 28, 377-401.

Powell, JL, Lewis, PA, Dunbar, RIM, Garcia-Finana, M, Roberts, N. 2010. Orbital prefrontal cortex volume correlate with social cognitive competence. Neuropsychologia, 48, 3554-3562.

Reader, SM, Hager, Y, Laland, KN. 2011. The evolution of primate general and cultural intelligence. Phil Trans R Soc B, 366, 1017-1027. 
Riccelli, R, Toschi, N, Nigro, S, Terracciano, A, Passamonti, L. 2017. Surface-based morphometry reveals the neuroanatomical basis of the five-factor model of personality. Soc Cogn Affect Neurosci, 12(4), 671-684.

Ripa, J, Storlind, L, Lundberg, P, Brown, JS. 2009. Niche co-evolution in consumer-resource communities. Evol Ecol Res, 11, 305-323.

Romme, IA, de Reus, MA, Ophoff, Ra, Kahn, RS, van den Heuvel, MP. 2017. Connectome dysconnectivity and cortical gene expression in patients with schizophrenia. Biol Psychiatry, 81(6), 495-502.

Ross, CA, Margolis, RL. 2018. Research Domain Criteria: Cutting edge neuroscience or Galen's hummors revisited? Mol Neuropsychiatry, 4(3), 158-163.

Ross, CA, Margolis, RL. 2018. Research Domain Criteria: Strengths, weaknesses, and potential alternatives for future psychiatric research. Mol Neuropsychiatry, 5(4), 218-236.

Roughgarden, J. 2012. The social selection alternative to sexual selection. Phil Trans R Soc B, 367, 2294-2303.

Rubinov, M, Sporns, O. 2010. Complex network measures of brain connectivity: Uses and interpretations. Neurolmage, 52(3), 1050-1060.

Ruff, CB, Trinkaus, E, Holliday, TW. 1997. Body mass and encephalization in Pleistocene Homo. Nature, 387, 173176.

Schaefer, A, Kong, R, Gordon, EM, Laumann, TO, Zuo, XN, Holmes, AJ, Eickhoff, SB, Yeo, BTT. 2018. Local-Global parcellation of the human cerebral cortex from intrinsic functional connectivity MRI. Cereb Cortex, 28(9), 3095-3114.

Schuppli, C, van Schaik, CP. 2017. Discovering the base of the iceberg: A new way to look at cultural repertoires in primates. Folia Primatol, 88, 13-131.

Shapley, LS. 1953. Stochastic games. Proc Natl Acad Sci, 39(19), 1095-1100.

Sheneman, L, Hintze, A. 2017. Evolving autonomous learning in cognitive networks. Sci Rep, 7(1), 16712.

Shi, DM, Levina, A, Noori, HR. 2019. Refined parcellation of the nervous system by algorithmic detection of hidden features within communities. Phys Rev E, 100(1), 012301.

Shulman, GL, Fiez, J, Corbetta, M, Buckner, RL, Miezin, FM, Raichle, M, Petersen, SE. 1997. Common blood flow changes across visual tasks: II. Decreases in cerebral cortex. J Cogn Neurosci, 9(5), 648-663.

Singh, KD, Fawcett, IP. 2008. Transient and linearly graded deactivation of the human default-mode etwork by a visual detection task. Neurolmage, 41(1), 100-112.

Smaers, JB, Soligo, C. 2013. Brain reorganization, not relative brain size, primarily characterizes anthropoid brain evolution. Proc R Soc B, 280(20130269).

Snider, J, Pillai, A, Stevens, CF. 2010. A universal property of axonal and dendritic arbors. Neuron, 66, 45-56.

Solan, E, Vieille, N. 2015. Stochastic games. Proc Natl Acad Sci, 112(45), 137743-13746.

Song, C, Schwarzkopf, DS, Rees, G. 2013. Variability in visual cortex size reflects tradeoff between local orientation sensitivity and global orientation modulation. Nat Comm, 4(2201), 1-10.

Sporns, O. 2013. Network attributes for segregation and integration in the human brain. Curr Opin Neurobiol, 23(2), 162-171.

Squire, LR, Wixted, JT. 2011. The cognitive neuroscience of human memory since HM. Annu Rev Neurosci, 34, 259288.

Striedter, GF. 2019. Variation across species and levels: Implications for model species research. Brain Behav Evol, 93(2-3), 57-69.

Taylor, JS, Raes, J. 2004. Duplication and divergence: The evolution of new genes and old ideas. Annu Rev Genet, $38,615-643$.

Teeter, CM, Stevens, CF. 2011. A general principle of neural arbor branch density. Curr Biol, 21, 2105-2108.

Tomasi, D, Volwok, ND. 2011. Functional connectivity hubs in the human brain. Neurolmage, 57(3), 908-917.

Tzourio-Mayer, N, Mazoyer, B. 2017. Variations of planum temporale symmetry with Heschl's gyri duplications and association with cognitive abilities: MRI investigation of 428 volunteers. Brain Struct Funct, 222(6), 27112726.

Ummels, M, Wojtczak, D. 2011. The complexity of Nash equilibria in stochastic multiplayer games. Log Meth Comput Sci, 7.

Valk, SL, Xu, T, Margulies, DS, Masouleh, SK, Paquola, C, Goulas, A, Kochunov, P, Smallwood, J, Yeo, BTT, Bernhardt, BC, Eickhoff, SB. 2020. Shaping brain structure: Genetic and phylogenetic axes of macro scale organization of cortical thickness. Preprint available on BioArXiv, doi: https://doi.org/10.1101/2020.02.10.939561. 
Van den Heuvel, MP, Fornito, A. 2014. Brain networks in schizophrenia. Neuropsychol Rev, 24(1), 32-48.

Van den Heuvel, MP, Scholtens, LH, de Lange, SC, Pijnenburg, R, Cahn, W, van Haren, NEM, Sommer, IE, Bozzali, M, Koch, K, Boks, MP, Repple, J, Pievani, M, Li, L, Preuss, TM, Rilling, JK. 2019. Evolutionary modifications in human brain connectivity associated with schizophrenia. Brain, 142(2), 3991-4002.

Van Essen, DC, Newsome, WT, Maunsell, JH. 1984. The visual field representation in striate cortex of the macaque monkey: Asymmetries, anisotropies, and individual variability. Vision Res, 29, 429-448.

Wang, H, Wang, B, Normoyle, KP, Jackson, K, Spitler, K, Sharrock, MF, Miller, CM, Best, C, Llano, D, Du, R. 2014. Brain temperature and its fundamental properties: A review for clinical neuroscientists. Front Neurosci, 8(307).

Waters, F, Jardrim R. 2015. Auditory hallucinations: Debunking the myth of language supremacy. Schizophr bull, 41(3), 533-534.

Weniger, G, Lange, C, Irle, E. 2006. Abnormal size of the amygdala predicts impaired emotional memory in major depressive disorder. J Affect Disord, 94, 219-229.

Whiten, A. 2017. How culture extends the scope of evolutionary biology in the great apes. Proc Natl Acad Sci, 114, 7790-7797.

Wilson, EO. 1978. On Human Nature. Harvard University Press.

Workman, AD, Charvet, CJ, Clancy, B, Darlington, RB, Finlay BL. 2013. Modeling transformations of neurodevelopmental sequences across mammalian species. J Neurosci, 33, 7368-7383. 